\title{
Inferences of Others' Competence Reduces Anticipation of Pain When under Threat
}

\author{
Ellen Tedeschi ${ }^{1}$, Jochen Weber ${ }^{1}$, Charlotte Prévost ${ }^{1,2}$, Walter Mischel ${ }^{1}$, and Dean Mobbs ${ }^{1}$
}

\begin{abstract}
On a daily basis, we place our lives in the hands of strangers. From dentists to pilots, we make inferences about their competence to perform their jobs and consequently to keep us from harm. Here we explore whether the perceived competence of others can alter one's anticipation of pain. In two studies, participants (Receivers) believed their chances of experiencing an aversive stimulus were directly dependent on the performance of another person (Players). We predicted that perceiving the Players as highly competent would reduce
\end{abstract}

\section{INTRODUCTION}

Evolutionary biologists have long speculated that one reason for group living is that it serves as a powerful survival strategy, whereby the company of others reduces the chances of being attacked by predators (Hamilton, 1971). Under such conditions of danger, the ability to detect protective traits in others (e.g., how brawny or how intelligent) is potentially adaptive (Zebrowitz \& Montepare, 2008). One of the fastest ways to evaluate these traits is through appearance, especially faces, and evidence suggests that these judgments are made automatically. Trait judgments based on faces made in a matter of milliseconds have been shown to be stable across time (Todorov, Pakrashi, \& Oosterhof, 2009). The detection of complex traits based on facial features alone is supported by research showing that judgments of competence predict real-world accomplishments. For example, when participants are shown faces of real election candidates, competence successfully predict electoral success (Mattes et al., 2010; Todorov, Mandisodza, Goren, \& Hall, 2005), and competence ratings of CEOs and law firm partners predicts both company and personal profits (Rule \& Ambady, 2008, 2011). These studies, however, have examined trait competence inconsequentially and without individuals making judgments in the context of direct consequence to themselves.

A few studies have examined competence perception in situations where it is directly relevant to the perceiver. For example, participants are more willing to disclose symptoms to physicians if they believed they were com-

${ }^{1}$ Columbia University, ${ }^{2}$ University of Geneva
Receivers' anxiety when anticipating the possibility of an electric shock. Results confirmed that high competence ratings consistently corresponded with lower reported anxiety, and complementary fMRI data showed that increased competence perception was further expressed as decreased activity in the bilateral posterior insula, a region localized to actual pain stimulation. These studies suggest that inferences of competence act as predictors of protection and reduce the expectation of negative outcomes. petent (Young, 1980), and the largest predictor of anxiety in dental patients during visits is the patients' perception of their dentist's technical competence (Rouse \& Hamilton, 1990). These studies suggest that competence may be part of a calculus that acts to predict and construct a likelihood of an aversive outcome (Fiske, Cuddy, \& Glick, 2006). A lingering question is whether the assessment of conspecifics' trait competence, especially when it confers their ability to protect us, alters the way we anticipate danger.

During the anticipation of pain, a network of wellcharacterized neural systems is evoked. This network includes the anterior and posterior insula (aINS and pINS, respectively), the ACC, the somatosensory cortex, and the amygdalae (Berns et al., 2006). Previous studies have found that the amount of activation in these regions correlates with levels of anticipation and dread of the painful stimuli (Drabant et al., 2011) and the individual's decreased confidence in the avoidance of the pain (Mobbs et al., 2007). Several of these same regions are also active during social judgments, suggesting that social factors may modulate this network during pain anticipation. Participants report less pain and show lower activation in ACC and insula during a painful stimulus when looking at a picture of their romantic partner than when looking at a stranger (Eisenberger et al., 2011) or holding the hand of a loved one (Coan, Schaefer, \& Davidson, 2006). Although these studies illustrate how social factors influence an individual's subjective experience of pain, no studies have examined how perceived competence of others mediates the anticipation of pain when others undertake a task with painful consequences to the perceiver. 
Here we investigate the relationship between perceptions of competence and the subjective appraisal of threat in a potentially painful situation. We created a task in which participants (Receivers) were told that their chance of experiencing an aversive stimulus (loud noise or electric shock) was directly dependent on whether another person (Player) made a mistake on a spatial and working memory task (SWMT) said to be related to competence. We predicted that the Receiver's perception of the Player's competence would mediate the anxiety they felt while waiting for a potential aversive noise (Study 1) and that activity in brain areas activated by painful electrical stimulation would decrease as a function of the perceived competence of the Player (Study 2).

\section{STUDY 1}

In our first experiment, we sought to establish a relationship between perceived competence and anxiety during a threat anticipation task. We began by creating and validating a set of photographs showing the faces of participants who played the SWMT. These pictures were independently rated for competence, intelligence, and trustworthiness and then were used in the creation of the task.

\section{Methods}

\section{Participants}

Participants were Columbia University students recruited from introductory psychology classes who received course credit for participation. A total of 128 individuals (84 women) participated in the three stages of Study 1. The Columbia University institutional review board reviewed and approved all procedures, and all participants provided written informed consent.

\section{Stimuli Creation and Validation}

Sixty participants ( 42 women) played a computerized SWMT and had their picture taken. Of the original 60, two were removed from further analysis because of unusable pictures. The SWMT tested abilities that are commonly included in measures of intelligence (Holdnack, Zhou, Larrabee, Millis, \& Salthous, 2011; Cain \& Allin, 2005), while also requiring players to learn quickly, make few mistakes, and handle increasing levels of difficulty. Each player played the game twice. They were video-recorded while playing and also had a picture of their face taken with a neutral expression. The pictures were cropped and edited to a standardized size and brightness.

A second group of participants ( $n=39,25$ women, 2 excluded for incomplete data) then rated the faces on their general competence, trustworthiness, and intelligence using 7-point Likert scales from 1 (not at all) to
7 (extremely). The raters were not told anything about the people shown in the pictures or about their performance on the SWMT. We did not find that performance on the SWMT was associated with any personality ratings made based on the players' faces. Nevertheless, ratings were highly consistent across raters, with many players being repeatedly rated as high or low in the three traits (competence: Cronbach's $\alpha=.938$; intelligence: $\alpha=.933$, trustworthiness: $\alpha=.895)$. The three traits were also highly correlated with each (competence/intelligence: $r(56)=$ $.757, p<.001$; competence/trustworthiness: $r(56)=.923$, $p<.001$; intelligence/trustworthiness: $r(56)=.787, p<$ $.001)$. However, we chose to continue with only the competence ratings (for further explanation, see Discussion).

The pictures were divided into three groups based on competence ratings. The low competence group ( $n=$ $15, M=3.38, S D=0.43$ ) was made up of all faces that were rated at least half a standard deviation below the mean $(4.31, S D=0.78)$, whereas the high competence group included faces at least half a standard deviation above the mean $(n=15, M=5.21, S D=0.34)$. The rest of the faces were placed in the midcompetence group $(n=28)$. These groups were used in Studies 1 and 2 to test whether high and low competence faces would elicit different levels of anxiety in participants who believed they were relying on the person in the picture to keep them safe.

\section{Competence and Anticipation of Pain Task}

We investigated whether the perception of competence influenced anxiety levels associated with threat anticipation. Participants ( $n=31,17$ women) were told that the people in the pictures (Players) had played a computer game that measured competence and that, if a player had made a mistake on the game, they (the Receiver) would hear a loud and unpleasant noise. Unbeknown to the Receivers, the noises were actually assigned to six pictures in the midcompetence group (three men and three women). The Receivers saw each face alone for 4 sec and then were given 6 sec to rate how competent they thought that Player was at the game on a Likert scale of 1-7. A 3-sec movie clip of the game starting was shown, followed by a black screen for a period of 10 $14 \mathrm{sec}$ when the game was supposed to occur. The aversive noise could occur at any time during this period. At the end of the anticipation period, Receivers rated how much anxiety they had felt while waiting on a Likert scale of 1 (not at all anxious) to 7 (extremely anxious; see Figure 1C). There were 58 total trials, with the aversive noise occurring in six of those trials. The six noises were all matched with Player faces that were rated in the midcompetence group during the validation stage.

The noise stimulus was the sound of a human scream, which lasted $750 \mathrm{msec}$. The sound was played through headphones worn by the participant and never exceeded $103 \mathrm{~dB}$. This level of sound is deemed aversive yet is not 


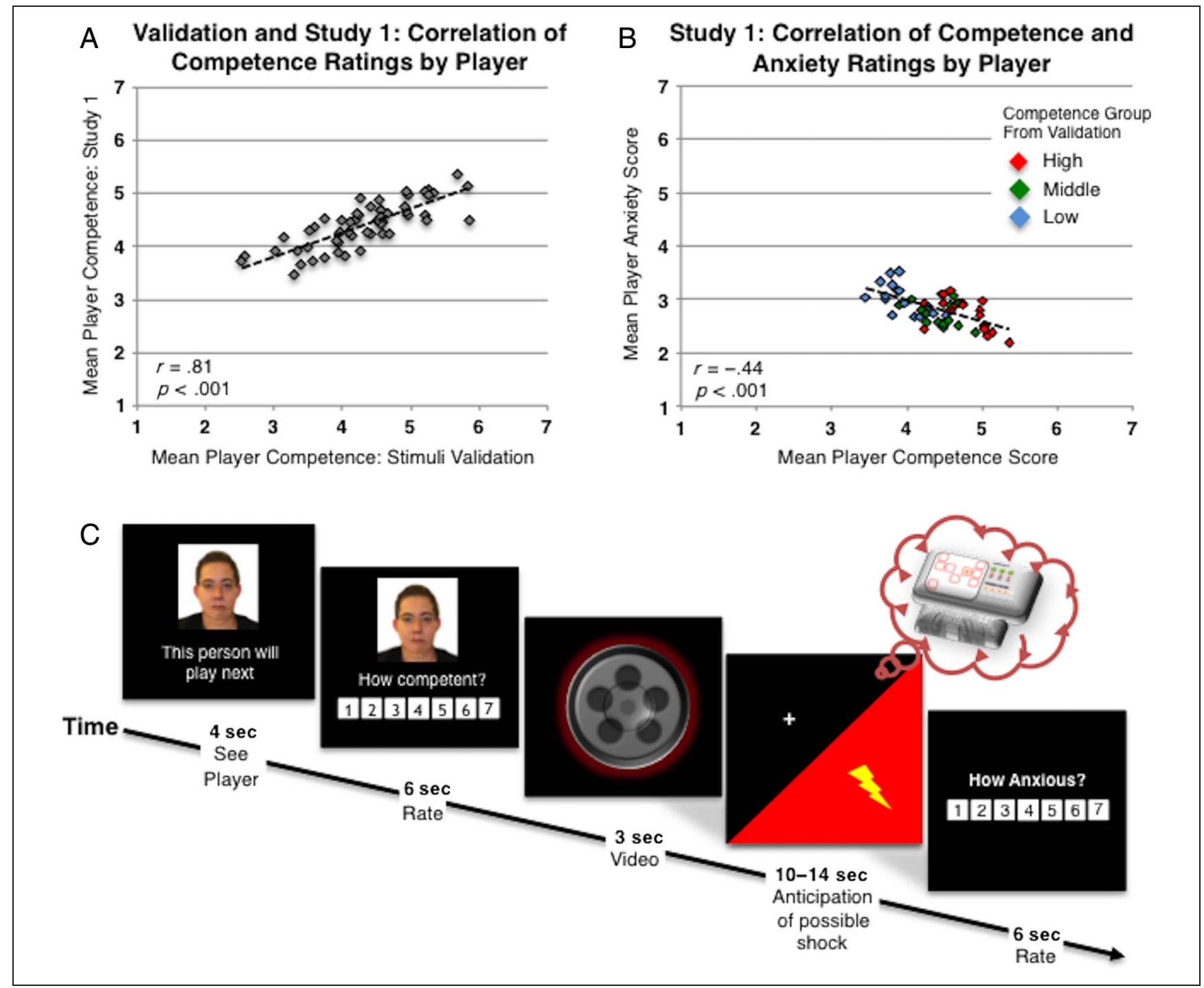

Figure 1. (A) Correlation between competence ratings from stimuli validation phase and Study 1 by Player picture. (B) Correlation between competence and anxiety ratings by Player picture during Study 1 . Colors show each Player's competence group from the validation phase.

(C) Temporal sequence of the paradigm for Studies 1 and 2: Participants view the face of one of the players of the SWMT task, rate them on competence, view a brief video of the beginning of the task, wait for an anticipation period in which a shock or noise might be delivered if the Player made a mistake, and then rate how much anxiety they felt during the anticipation period.

loud enough to cause any temporary or permanent damage. The stimuli was calibrated before the start of the task; participants were first exposed to the stimulus at a low volume, and then the experimenter increased the volume incrementally until the participant reported that it was uncomfortable but not unbearable or until the maximum threshold was reached. Participants were exposed to the stimulus three to five times during the calibration.

\section{Results}

For this analysis, ratings were averaged across participants resulting in a competence and anxiety score for each of the Player pictures. The competence ratings from Study 1 were highly correlated with those from stimuli validation $(r(56)=.79, p<.001$; see Figure $1 \mathrm{~A})$ and again showed high agreement between raters $(\alpha=$ .789), further supporting the conclusion that competence perceptions are consistent across individuals. Importantly, higher Player competence ratings were correlated with lower reported anxiety during the anticipation period $(r(56)=-.62, p<.001$; see Figure $1 \mathrm{~B})$, and anxiety scores were significantly lower for the high competence picture group $(M=2.71, S D=0.30, n=$ 15) than the low competence group $(M=3.04, S D=$ $0.28, n=15 ; t(28)=3.16, p=.004, d=1.14)$.

\section{STUDY 2}

In Study 2, we modified the task from Study 1 for fMRI to replicate the behavioral findings and gather complementary 
physiological evidence. The main goal of the study was to examine the neural correlates of competence perception and anticipation of aversive stimuli, specifically the decrease in reported anxiety during the anticipation period.

\section{Methods}

\section{Participants}

Participants ( $n=21,11$ women) were neurologically healthy, right-handed individuals with no history of heart problems and normal or corrected-to-normal vision recruited from the Columbia University community. All procedures were approved by the Columbia University institutional review board. Participants were paid for their involvement and provided informed written consent. We set an intended sample size of 20, based on standard practices for fMRI research.

\section{Procedures}

In this study, the task from Study 1 was modified for use in an MRI scanner, and the aversive noise was replaced with an electric shock. The task was divided into three blocks. Two of the blocks (20 trials) contained three shocks each, although participants were not informed beforehand of the number of shocks that would occur. The other block (18 trials) contained no shocks, but instead the screen would turn red three times during the anticipation period. Participants were told that this indicated that a shock would have occurred. Participants were told at the beginning of each block if the block contained shocks. The Player faces were arranged so that all the faces that fell into the high or low competence groups ( $n=15$ each) were placed into the two shock blocks. The no-shock block and the remaining faces in each shock block were midcompetence faces (including the faces paired with shocks).

In this study, the aversive stimulus was a shock rather than a noise, as we felt that the noise would lose its effectiveness when competing with the sound of the MRI scanner. The shock stimuli were delivered using a Biopac MP150 with an STM100C module (Biopac Systems, Inc., Goleta, CA). Attached to the STM100C was a 200-V maximum stimulus isolation unit (STMISOC, Biopac Systems). Shocks were administered via pregelled radiotranslucent electrodes on the underside of the participant's left wrist and attached to the STMISOC with shielded leads. Shock calibration followed the same process as the aversive noise, with an upper limit of $100 \mathrm{~V}$.

\section{Imaging Acquisition}

MRI scanning took place at the Neurological Institute at Columbia University Medical Center, using a 1.5-T GE Twin Speed MRI scanner. The functional images were gradient-echo, echoplanar T2*-weighted images (EPI) acquired in an interleaved order with BOLD contrast. Each volume was composed of 28 axial slices ( $4 \mathrm{~mm}$ thickness) aligned along the axis between the anterior and posterior commissures $(64 \times 64$ voxels, $3.5 \times$ $3.5 \mathrm{~mm}$ in-plane resolution, field of view $=224 \mathrm{~mm}$, repetition time $=2000 \mathrm{msec}$, echo time $=34 \mathrm{msec}$, flip angle $=84^{\circ}$ ). Each session consisted of three 11-min functional runs with volumes acquired continuously every 2 sec. The first three volumes of each run were discarded from further analysis to allow for T2* contrast stabilization. A T1-weighted structural scan was acquired at the end of the session.

\section{Preprocessing}

Structural images were subjected to the Unified Segmentation algorithm implemented in SPM8, yielding discrete cosine transform spatial warping coefficients used to normalize (warp) each individual's data (structural and functional) into ICBM-152 (MNI) space.

Functional data were preprocessed using the following SPM8 functions: slice-time correction, two-pass realignment to correct for head motion (rigid body registration of all frames to the averaged image after first pass), coregistration of each participant's functional mean image to the corresponding structural image, followed by applying the normalization parameters determined during segmentation to the functional images, and then using a 6-mm FWHM Gaussian smoothing kernel.

\section{Whole-brain Analysis}

Preprocessed images were subjected to a two-level general linear model using NeuroElf v0.9d (neuroelf.net/). The first (individual participant) level contained the following regressors of interest, which were all convolved with the canonical two-gamma hemodynamic response function: a 2-sec box-car function for the stimulus (face) period, a 6-sec box-car function for the competency rating period, and a 10-to-14-sec (duration-jittered) box-car function for the anticipation period, followed by a 6-sec box-car function for the anxiety rating period. In addition, an orthogonal regressor using the mean-removed competency ratings parametrically modulating the anticipation period was used. Furthermore, regressors of no interest consisted of motion parameters determined during preprocessing, their first temporal derivative, and discrete cosine transform-based temporal low-frequency drift regressors with a cutoff of 192 sec. Beta (regression weight) maps were used to create linear contrast maps (weighted sums of betas), which were then subjected to several second-level, random-effects (summary statistics) one-sample $t$ tests, with the NULL hypothesis being that the mean over all participants is zero (0). Our model included the face-viewing period ( $4 \mathrm{sec}$ ), the competence rating (6 sec), the anticipation period (10-14 sec), and 
the anxiety rating $(6 \mathrm{sec})$. The main analyses focused on the 10-14 sec anticipation period during each trial when a shock did not occur.

The resulting statistical maps (one-sample $t$ tests and a conjunction of two tests) were thresholded using a combination of uncorrected alpha levels together with a cluster size threshold determined by Monte Carlo simulations implemented in AlphaSim. Taken together, the two thresholds for each map provide a whole-brain FWE-correction threshold of $p<.05$; that is, the combined $\mathrm{p}$ - and k-thresholds given in the Results section signify whole-brain corrected FWE $p<.05$ results. For each contrast's $t$ map, AlphaSim uses the estimated, spatially averaged smoothness of the residual from the secondlevel regression. Additionally, for results with a strong a priori spatial hypotheses, a small volume correction (SVC) was applied by dividing the uncorrected desired false-positive level $(5 \%, p<.05)$ by the number of estimated resels in the search, yielding a corrected threshold (Denny, Ochsner, Weber, \& Wager, 2014; Zaki, Weber, \& Ochsner, 2012).

\section{Results}

Behavioral results replicated our previous findings: agreement between raters on the competence or incompetence of the faces was high $(\alpha=.766)$, and competence ratings were positively correlated with those from the stimuli validation $(r(56)=.82, p<.001)$ and Study $1(r(56)=.80, p<.001)$. As in Study 1, competence ratings (by Player) were negatively correlated with anxiety ratings during the anticipation period $(r(32)=$ $-.44, p<.01$; shock trials removed). However, this was only true in the blocks where a shock was possible. No correlation was found between competence and anxiety ratings in the separate control block in which participants were told that there would be no shocks (only a red screen) when a mistake occurred $(r(15)=.078$, $p=.782)$. A formal interaction test was not conducted given the nature of the samples, because the Players in the no-shock block were all previously rated as midcompetence, and all high and low competence faces were placed in the shock blocks. However, the difference in the polarity of the $r$-values suggests that this difference is robust. Although this may be because of a floor effect for anxiety ratings in the no-shock condition, this is consistent with our hypotheses, as Receivers should have no reason to feel anxiety in the absence of a threat. Anxiety scores in the no-shock condition were significantly lower $(M=2.66, S D=0.25)$ than in the shock condition $(M=$ $3.64, S D=0.31 ; t(56)=-11.84, p<.001)$. There was no difference in competence scores between the two conditions $(t(56)=.763, p=.45)$.

In the fMRI data analysis, we focused on the anticipation period. In the first contrast, we examined the direct effects of receiving a shock by comparing activity during the 3 -sec window immediately following a shock (six tri- als) with the same window from the red screen trials in the control block, when participants were told a shock would have occurred (three trials). This contrast showed increased activity throughout the pain matrix for the shock compared to the red screen, especially the bilateral pINS (Figure 2, red bar; RH: 36, $-18,6, t=7.375$; LH: $-36,-24,6, t=5.274$; voxel-wise $p<.02, k=324$, FWE $p<.05$, estimated smoothness $=11.6 \mathrm{~mm}$ ).

In the next contrast, we analyzed the effects of pain anticipation in trials when a shock did not occur. For this, we looked at the entire anticipation period in trials when there was a possibility of a shock, but a shock did not occur (34 trials), using Receivers' competence ratings as a trial-by-trial parametric regressor. This showed reduced activity in the bilateral insula in response to high competence ratings compared to low competence ratings (Figure 2, blue bar; RH: 36, $-18,9, t=-3.921$; LH $-33,-18,3, t=-4.104$; voxel-wise $p<.02, k=$ 469 , FWE $p<.05$, estimated smoothness $=13.7 \mathrm{~mm}$ ).

Visually, the areas of activation for these two contrasts (actual pain and anticipated pain moderated by competence) showed extensive overlap. We conducted a conjunction analysis $(p<.02, k=32$, estimated smoothness $=$ $11.6 \mathrm{~mm}$; combined threshold estimated simulating the conjunction of two independent maps), which showed that the areas of overlap in the bilateral pINS (RH: peak 37, $-22,12, k=744$; LH: peak $-37,-17,7, k=299$ ) were statistically significant (Figure 2B).

To further test this effect, we also conducted an ROI analysis on the parametric competence contrast using the peak coordinates of the pain-related activity in the bilateral insula as localizers (two 5-mm diameter spheres in left and right pINS at peaks from the shock $>$ red screen contrast). This resulted in two clusters in the pINS, again showing that when Receivers rated the Players as higher in competence, there was a parametric decrease in activity. The activation was strongest in the right pINS (37, $-22,10 ; t(20)=3.92, p=.01, k=10, \mathrm{SVC}$ ), whereas the activation in the left pINS was less robust, but still significant $(-37,-19,9 ; t(20)=-3.58, p=.02, k=3, \mathrm{SVC})$.

Conversely, in the no-shock condition (15 trials, red screen trials removed), there was no significant areas of activation in response to parametric competence. The

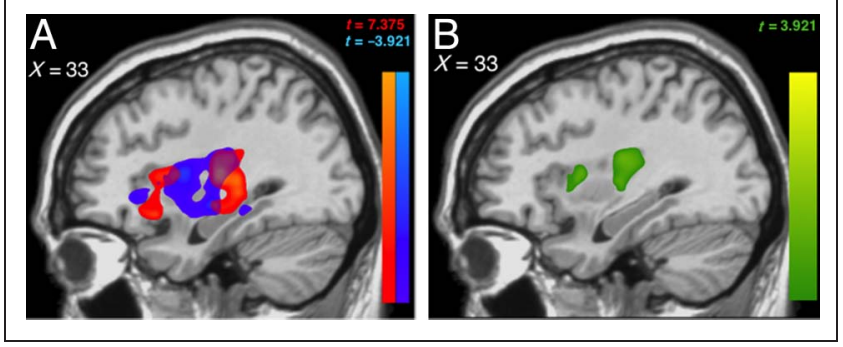

Figure 2. (A) Overlap between right pINS activity associated with shock pain (red) and decreased activity associated with increased parametric ratings of Player competence (blue). (B) Conjunction analysis performed between contrasts shown in (A). Image threshold $p<.02$. 
observed statistic for the parametric modulator of competence during the waiting period was nonsignificant (left side at peak: $t=-0.224, p>.1$; right side peak: $t=0.955, p>.1)$. This is consistent with the behavioral results and suggests that competence was only important to Receivers when under threat of pain.

Although we measured Receivers' reported anxiety behaviorally, a separate model using parametric anxiety ratings rather than competence ratings produced few areas of activation, none of which survived correction. Even at the lowest thresholds, there was no activity in any a priori area of interest in response to parametric anxiety.

\section{DISCUSSION}

Danger often occurs when we are in the presence of others, and during these situations, we may look to them for protection. Our findings demonstrate that perceived competence based on others' facial features works to reduce anticipation of pain when under threat. Overall, the results of two studies support the hypothesis that perceived competence of others can influence one's feelings of anxiety and pain anticipation. In Study 1, participants' reported anxiety levels were reduced when they predicted Players to be competent at the SWMT. In Study 2, we replicated the behavioral findings of Study 1 and found that, during anticipation of the aversive stimulus, activity in areas of the bilateral pINS localized to pain were down-regulated by the perceived competence of the Player.

The behavioral findings of Studies 1 and 2 expand upon previous research on perceived competence. As in prior work, participants were able to quickly judge the Players' competence. However, instead of predicting election results or profits (Rule \& Ambady, 2008; Todorov et al., 2005), competence ratings predicted the Receivers' own feelings of anxiety. Spezio et al. (2008) found that, although competence ratings predicted election outcomes, ratings of personal threat did so as well. If facial signals that convey low competence also communicate a potential threat, this may help to explain the increases in anxiety when faced with an incompetent Player. It would be evolutionarily advantageous to perceive incompetence as a signal of potential danger, because being in a group with incompetent individuals could lead to decreased safety for all. It is important to note that this effect cannot be attributed simply to an increase in anxiety, as the parametric anxiety ratings elicited no significant areas of activation during the anticipation period. This suggests that perceived competence is affecting participants' predictions about the possibility of the unpleasant stimulus.

In a task such as ours, this effect is especially relevant, because the Receivers' potential discomfort was presented as directly dependent on the skills of the Players. Although their ratings were not related to actual abilities, perceiving a Player to be highly competent reduced their reported anxiety during the pain anticipation. This is in line with previous work on trait perception, specifically trustworthi- ness. A study that examined cooperative behavior during trust games found that perceptions of trustworthiness were used to predict the probability of reciprocation during interactions with partners (Chang, Doll, van't Wout, Frank, \& Sanfey, 2010). Overall, this suggests that individuals use trait perceptions as a way to predict the behavior of others and then modify their actions and expectations according to these predictions.

By comparing trial-by-trial parametric competence modulation with the actual effects of the painful stimulus, the complementary fMRI data from Study 2 show pronounced activity in the pINS during the periods when participants were anticipating pain. Research has shown that the insula is involved in a broad range of cognitive, emotional, and physical processes. However, it has been consistently divided into functional subregions (Chang, Yarkoni, Khaw, \& Sanfey, 2013). The ventroanterior and dorsoanterior regions are involved in emotional and cognitive processes, whereas the pINS is activated mainly by physiological sensations, particularly pain (Chang et al., 2013; Craig, 2002). Craig (2009) proposed that activity related to introspective awareness begins in the pINS with basic physiological input and progresses along an anterior-posterior flow to the aINS. The insula receives input from other regions at different stages along this progression, integrating environmental, emotional, and social contexts into a complete sense of awareness. In this model, pain signals start in the pINS, but factors such as attention, anxiety, and social cues are integrated as the signal progresses to the aINS, changing how pain is experienced (Eisenberger et al., 2011; Simmons et al., 2011; Simmons, Matthews, Stein, \& Paulus, 2004; Bantick et al., 2002).

Evidence suggests the neural circuitry that responds to pain, which includes the pINS, also shapes how we anticipate and interpret to pain. Berns et al. (2006) found that the pINS and other areas of the pain circuitry were activated during anticipation of a recurrent shock. The effect was especially strong in participants labeled as "extreme dreaders": those who, when given a choice, preferred to receive a shock sooner rather than later, even if it meant a higher voltage, to avoid extended waiting. As in our study, this activation was found in the same areas as the activation caused by the shock, suggesting that dreading a painful stimulus activates the same network as the pain itself.

Extensive research has shown that pain is not a purely physiological sensation, and expectations can change how it is experienced. When individuals expect to feel only a small amount of pain, they rate a subsequent stimulus as less painful than they otherwise would, even if the intensity is actually the same (Brown, Seymour, Boyle, El-Deredy, \& Jones, 2008; Wager et al., 2004). Conversely, those who expect to feel a large amount of pain may rate even a nonpainful stimulus as painful (Sawamoto et al., 2000). In both cases, brain activity in the pain matrix has been shown to reflect one's expectations. Drabant 
et al. (2011) even found that regions of the insula and ACC responded to anticipated pain intensity in a stepwise pattern, where the highest activation occurred when the largest shock intensity was predicted. This shows that the brain's pain matrix may be sensitive to the probability of experiencing pain and that specific expectations can modulate its response. In Study 2, it is therefore possible that competence was used to predict the chance of experiencing pain. Perceived competence may be used as a type of "probability of pain" analysis, in the same way that nonsocial information has been used in previous studies. That is, upon seeing a high competence Player, Receivers may have judged the probability of receiving shock in that trial as low, resulting in low anxiety and decreased activation in the pINS.

Across both studies and the stimuli validation, we found no relationship between SWMT performance and independent competency ratings of the Players' faces. One possible explanation for this result may be that despite the SWMT's predicted connection to IQ, strategic thinking, and problem-solving (Sternberg, 2005), it may not have been an ideal instrument to test the connection between competency and physiognomy. Another possibility is that the static, neutral faces of the players did not provide sufficient information to form an accurate impression of competence. Although prior research on face perception shows that trait ratings can be made for neutral faces in as little as a few milliseconds (Todorov et al., 2009), studies that have successfully tied ratings of personality traits to accuracy have either used more complex interactions, such as handshakes (Chaplin, Phillips, Brown, Clanton, \& Stein, 2000), or have produced relatively small effects that held for some traits, but not for others (Todorov et al., 2009). In the real world, it is likely that dynamic movement of faces, along with body language, dress, and vocal inflection strengthen the accuracy of impressions. However, raters' impressions were highly consistent, and so regardless of its reflection of actual ability, there seems to be a stereotypical template that embodies competence. This perception, regardless of its accuracy, was enough to affect the predictions and expectations of the Receivers regarding their own risk of pain.

During the stimulus validation, we found that competence ratings were highly correlated with both trustworthiness and intelligence ratings. It is therefore possible that either of these could be driving the reduction in pain anticipation, rather than competence. However, we chose to focus our attention on competence based on previous findings in the literature regarding the interactions of these traits. In the case of trustworthiness, several studies on competence and election results have shown that competence ratings predict those results far better than ratings related to trustworthiness (Mattes et al., 2010; Spezio et al., 2008; Todorov et al., 2005). In addition, Receivers would have no reason to use trustworthiness as an indicator for the probability of the un- pleasant stimulus in this task, as the Players had no idea that their performance could result in a negative outcome for anyone else. As for intelligence, research on trait perception suggests that competence and intelligence are highly interconnected. Todorov et al. (2005) conducted a factor analysis of a variety of traits possibly related to the prediction of election results and found that competence and intelligence ratings clustered into one factor, which had more predictive power than a separate factor related to trustworthiness. Competence is thought to reflect an overlapping set of skills, including intelligence, motivation, strategic thinking, and problem solving (Sternberg, 2005). We felt that using competence, in the context of rating the protective qualities of another individual, was more relevant than intelligence.

Our two studies illustrate that people use assessments of the competence of others to inform their reactions when anticipating potential negative events. Perception of high competence leads to a reduction of the anxious state as well as down-regulation of the neural systems that underlie the preparation for forth-coming pain. More speculatively, inferred competence in another's ability to protect us may reduce stress associated with chronic threat and may be a trait that is optimized to secure intragroup value by helping others to survive. Concerning the current utility of inferred competence, these findings underscore its importance in reducing negative effect in a variety of professions, including how physicians ease anxiety in their patients and how this facilitates patient care and therapeutic efficacy across all domains of medicine.

Reprint requests should be sent to Ellen Tedeschi, Fear, Anxiety and Biosocial Behavioral Laboratory, Department of Psychology, Columbia University, 406 Schermerhorn Hall, 1190 Amsterdam Ave. MC 5501, New York, NY 10027, or via e-mail: eer2135@columbia.edu, ellentedeschi@gmail.com.

\section{REFERENCES}

Bantick, S. J., Wise, R., Ploghaus, A., Clare, S., Smith, S., \& Tracey, I. (2002). Imaging how attention modulates pain in humans using functional MRI. Brain, 125, 310-319.

Berns, G., Chappelow, J., Cekic, M., Zink, C., Pagnoni, G., \& Martin-Skurski, M. (2006). Neurobiological substrates of dread. Science, 312, 754-758.

Brown, C. A., Seymour, B., Boyle, Y., El-Deredy, W., \& Jones, A. K. (2008). Modulation of pain ratings by expectation and uncertainty: Behavioral characteristics and anticipatory neural correlates. Pain, 135, 240-250.

Cain, S., \& Allin, J. (2005). Book review: Stanford-Binet Intelligence Scales, fifth edition. Journal of Psychoeducational Assessment, 23, 87-95.

Chang, L. J., Doll, B. B., van 't Wout, M., Frank, M. J., \& Sanfey, A. G. (2010). Seeing is believing: Trustworthiness as a dynamic belief. Cognitive Psychology, 61, 87-105.

Chang, L. J., Yarkoni, T., Khaw, M. W., \& Sanfey, A. (2013). Decoding the role of the insula in human cognition: Functional parcellation and large-scale reverse inference. Cerebral Cortex, 23, 739-749. 
Chaplin, W. F., Phillips, J., Brown, J., Clanton, N., \& Stein, J. (2000). Handshaking, gender, personality, and first impressions. Journal of Personality and Social Psychology, 79, 110-117.

Coan, J. A., Schaefer, H. S., \& Davidson, R. J. (2006). Lending a hand: Social regulation of the neural response to threat. Psychological Science, 17, 1032-1039.

Craig, A. D. (2002). How do you feel? Interoception: The sense of the physiological condition of the body. Nature Reviews Neuroscience, 3, 655-666.

Craig, A. D. (2009). How do you feel-Now? The anterior insula and human awareness. Nature Reviews Neuroscience, 10, 59-70.

Denny, B. T., Ochsner, K. N., Weber, J., \& Wager, T. D. (2014). Anticipatory brain activity predicts the success or failure of subsequent emotion regulation. Social Cognitive and Affective Neuroscience, 9, 403-411.

Drabant, E., Kuo, J., Ramel, W., Blechert, J., Edge, M., Cooper, J., et al. (2011). Experiential, autonomic, and neural responses during threat anticipation vary as a function of threat intensity and neuroticism. Neuroimage, 55, 401-410.

Eisenberger, N., Master, S., Inagaki, T., Taylor, S., Shirinyan, D., Lieberman, M., et al. (2011). Attachment figures activate a safety signal-related neural region and reduce pain experience. Proceedings of the National Academy of Sciences, U.S.A., 108, 11721-11726.

Fiske, S., Cuddy, A., \& Glick, P. (2006). Universal dimensions of social cognition: Warmth and competence. Trends in Cognitive Science, 11, 77-83.

Hamilton, W. D. (1971). Geometry of the selfish herd. The Journal of Theoretical Biology, 31, 295-311.

Holdnack, J. A., Zhou, X., Larrabee, G., Millis, S., \& Salthous, T. (2011). Confirmatory factor analysis of the WAIS-IV/WMS-IV. Assessment, 18, 178-191.

Mattes, K., Spezio, M., Kim, H., Todorov, A., Adolphs, R., \& Alvarez, R. (2010). Predicting election outcomes from positive and negative trait assessments of candidate images. Political Psychology, 31, 41-58.

Mobbs, D., Petrovic, P., Marchant, J., Hassabis, D., Weiskopf, N., Seymour, B., et al. (2007). When fear is near: Threat imminence elicits prefrontal-periaqueductal gray shifts in humans. Science, 317, 1079-1083.

Rouse, R., \& Hamilton, M. (1990). Dentists' technical competence, communication and personality as predictors of dental patient anxiety. Journal of Behavioral Medicine, 13, 307-319.
Rule, N. O., \& Ambady, N. (2008). The face of success: Inferences from chief executive officers' appearance predict company profits. Psychological Science, 19, 109-111.

Rule, N. O., \& Ambady, N. (2011). Face and fortune: Inferences of personality from managing partners' faces predict their law firms' financial success. Leadership Quarterly, 22, 690-696.

Sawamoto, N., Honda, M., Okada, T., Hanakawa, T., Kanda, M., Fukuyama, H., et al. (2000). Expectation of pain enhances responses to nonpainful somatosensory stimulation in the anterior cingulate cortex and parietal operculum/posterior insula: An event-related functional magnetic resonance imaging study. Journal of Neuroscience, 20, 7438-7445.

Simmons, A. N., Matthews, S., Stein, M., \& Paulus, M. (2004). Anticipation of emotionally aversive visual stimuli activates right insula. NeuroReport, 15, 2261-2263.

Simmons, A. N., Stein, M., Strigo, I., Arce, E., Hitchcock, C., \& Paulus, M. (2011). Anxiety positive subjects show altered processing in the anterior insula during anticipation of negative stimuli. Human Brain Mapping, 32, 1836-1846.

Spezio, M. L., Rangel, A., Alvarez, R., O'Doherty, J., Mattes, K., Todorov, A., et al. (2008). A neural basis for the effect of candidate appearance on election outcomes. Social Cognitive and Affective Neuroscience, 3, 344-352.

Sternberg, R. J. (2005). Intelligence, competence, and expertise. In A. Elliot \& C. Dweck (Eds.), Handbook of competence and motivation (pp. 15-30). New York: Guilford Press.

Todorov, A., Mandisodza, A., Goren, A., \& Hall, C. (2005). Inferences of competence from faces predict election outcomes. Science, 308, 1623-1626.

Todorov, A., Pakrashi, M., \& Oosterhof, N. (2009). Evaluating faces on trustworthiness after minimal time exposure. Social Cognition, 27, 813-833.

Wager, T. D., Rilling, J. K., Smith, E. E., Sololik, A., Casey, K. L., Davidson, R. J., et al. (2004). Placebo-induced changes in fMRI in the anticipation and experience of pain. Science, 202, $1162-1167$.

Young, J. W. (1980). The effects of perceived physician competence on patients' symptom disclosure to male and female physicians. Journal of Behavioral Medicine, 3, 279-290

Zaki, J., Weber, J., \& Ochsner, K. (2012). Task-dependent neural bases of perceiving emotionally expressive targets. Frontiers in Human Neuroscience, 6, 1-11.

Zebrowitz, L. A., \& Montepare, J. (2008). Social psychological face perception: Why appearance matters. Social and Personality Psychology Compass, 2, 1497-1517. 